\title{
Thrombolysis during extended cardiopulmonary resuscitation for autoimmune-related pulmonary embolism
}

\author{
Jian-ping Gao ${ }^{1}$, Ke-jing Ying ${ }^{2}$ \\ ${ }^{1}$ Department of Emergency Medicine, Sir Run Run Shaw Hospital, Zhejiang University School of Medicine, Hangzhou \\ 310016, China \\ ${ }^{2}$ Department of Respiratory and Critical Care, Sir Run Run Shaw Hospital, Zhejiang University School of Medicine, \\ Hangzhou 310016, China
}

Corresponding Author: Ke-jing Ying, Email: zrjzk@zju.edu.cn

BACKGROUND: Massive pulmonary embolism (MPE) and acute myocardial infarction are the two most common causes of cardiac arrest (CA). At present, lethal hemorrhage makes thrombolytic therapy underused during cardiopulmonary resuscitation, despite the potential benefits for these underlying conditions. Hypercoagulability of the blood in autoimmune disorders (such as autoimmune hemolytic anemia) carries a risk of MPE. It is critical to find out the etiology of CA for timely thrombolytic intervention.

METHODS: A 23-year-old woman with a 10-year medical history of autoimmune hemolytic anemia suffered from CA in our emergency intensive care unit. ECG and echocardiogram indicated the possibility of MPE, so fibrinolytic therapy (alteplase) was successful during prolonged resuscitation.

RESULTS: Neurological recovery of the patient was generally good, and no fatal bleeding developed. MPE was documented by CT pulmonary angiography.

CONCLUSIONS: A medical history of autoimmune disease poses a risk of $\mathrm{PE}$, and the causes of CA (such as this) should be investigated etiologically. A therapy with alteplase may be used early during cardiopulmonary resuscitation once there is presumptive evidence of PE. Clinical trials are needed in this setting to study patients with hypercoagulable states.

KEY WORDS: Pulmonary embolism; Thrombolysis; Cardiac arrest; Autoimmune disease

World J Emerg Med 2015;6(2):153-156

DOI: 10.5847/wjem.j.1920-8642.2015.02.012

\section{INTRODUCTION}

The prognosis of patients with cardiac arrest (CA) is dismal, whether or not they are hospitalized, with survival rates about $15 \%$ and $8 \%$, respectively. These figures are even worse if nearly a full recovery (i.e. no irreversible neurological impairment) is stipulated. CA is commonly attributable to thromboembolic diseases, such as massive pulmonary embolism (MPE) and acute myocardial infarction (AMI). Autoimmune diseases, particularly autoimmune hemolytic anemia (AIHA), are associated with blood hypercoagulability, so care must be taken to prevent fatal thromboembolism. ${ }^{[1]}$

During cardiopulmonary resuscitation (CPR), systemic thrombolysis is a controversial therapeutic approach due to potentially lethal hemorrhage. Current advanced cardiac life support (ACLS) guidelines maintain that evidence is still insufficient to recommend or discourage routine use of thrombolytic drugs during CPR, although empiric fibrinolytic therapy may be considered if PE is implicated. ${ }^{[2]}$

Reported herein is a successful resuscitation, aided by the fibrinolytic agent alteplase (recombinant tissue 
plasminogen activator). Based on presumptive evidence of MPE (due to AIHA), treatment was given during the extended CPR, which was initiated after a documented CA occurred in our emergency intensive care unit (EICU). This scenario highlights the importance of medical history for CPR procedures in terms of blood hypercoagulability.

\section{CASE REPORT}

A 23-year-old woman presented to our emergency department (ED) with complaints of chills and fever for two days after being caught in the rain. She vomited twice (with melena), experiencing chest discomfort and light-headedness but no coughing, expectoration, or abdominal pain. Her vital signs were as follows: temperature $38.3{ }^{\circ} \mathrm{C}$; heart rate (HR) 135 beats/minute, respiratory rate 25 beats/minute, and BP $122 / 81 \mathrm{mmHg}$. In addition, she claimed a 10-year history of AIHA (untreated at the time) and a 3-year history of bronchial asthma, treated with Seretide. She denied smoking and alcohol abuse and had no history of hypertension or diabetes.

Physical examination revealed tachycardia, tachypnea, and shortness of breath, without crackles or wheeze. No abdominal tenderness, rebound tenderness, or rigidity was evident, and the lower extremities were not overtly edematous. Her chest X-ray was normal. Ultrasound examination of both upper and lower

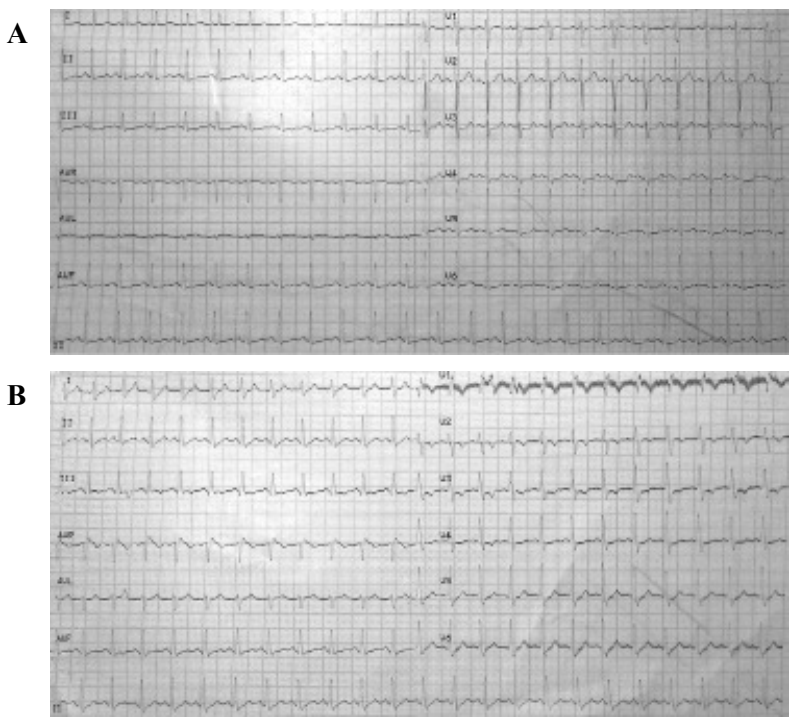

Figure 1. ECG (12 leads). A: ECG (12 leads) at the emergency department, displaying sinus tachycardia (HR 148 beats/minute); B: ECG (12 leads) recorded briefly before cardiac arrest, showing sinus tachycardia, incomplete right bundle-branch block, and $\mathrm{T}$ inversion in precordial leads V1-4. extremities showed no evidence of deep vein thrombosis (DVT), despite a moderate risk determined by Well's criteria for PE. Sinus tachycardia (HR 148 beats/minute) was noted on ECG (Figure 1A).

Initial blood analysis in ED showed the following: hemoglobin $67 \mathrm{~g} / \mathrm{L}$, reticulocytes $6 \%$, white cell count $39 \times 10^{9} / \mathrm{L}$, neutrophils $73.8 \%$, platelet count $224 \times 10^{9} / \mathrm{L}$, APTT 45 seconds, INR 1.5, D-dimer $9.8 \mu \mathrm{g} / \mathrm{mL}$ (normal range $0-0.5 \mu \mathrm{g} / \mathrm{mL}$ ), C-reactive protein $101.2 \mathrm{mg} / \mathrm{L}$, procalcitonin $1.2 \mathrm{ng} / \mathrm{mL}$, and lactic acid $3.5 \mathrm{mmol} / \mathrm{L}$. Direct and indirect Coombs tests (anti-IgG) were positive, and occult blood testing of the feces was markedly positive. ABG on a nasal tube with oxygen flow at $5 \mathrm{~L} / \mathrm{min}$ showed a $\mathrm{PaO}_{2} / \mathrm{FiO}_{2}$ of $250 \mathrm{mmHg}$.

The patient was admitted into an EICU, with primary diagnoses of suspected PE, AIHA, and severe sepsis. About 4 hours later, her BP and HR dropped dramatically in a matter of minutes, resulting in sudden CA. CPR started in a timely manner. A 12-lead ECG recorded before CA demonstrated sinus tachycardia, with incomplete right bundle-branch block and $\mathrm{T}$ inversion in precordial leads V1-4 (Figure 1B). Echocardiogram displayed right ventricular dilatation and moderate pulmonary hypertension, without pericardial effusion.

After a 20-minute period of consistent CPR, a refractory pulseless electrical activity (PEA) occurred. MPE or AMI was considered to potentially explain ECG and echocardiographic findings, so a thrombolytic agent was elected. A 5-mg bolus of alteplase was delivered intravenously, followed by a continuous infusion (45 $\mathrm{mg}$ ) over the next hour. The dosage was based on body weight and potential bleeding risk. CPR was continued for another 55 minutes, until the return of spontaneous circulation was achieved. After 4 hours of observation (to assure BP and cardiac rhythm/rate stabilization), CT pulmonary angiography (CTPA) and cerebral CT were done. MPE was apparent bilaterally (Figure 2) without obvious intracranial hemorrhage. The patient regained consciousness 6 hours after successful resuscitation. No neurologic deficit was evident, nor was the existing gastrointestinal hemorrhage aggravated. Unfortunately, she died of the multiple organ dysfunction induced by severe tissue infection 5 weeks later.

CA is generally attributable to hypovolemia, hypoxia, hydrogen ion acidosis, hypo-/hyperkalemia, and hypothermia (5 H's) or tension pneumothorax, tamponade, cardiac toxins, pulmonary thrombosis, and coronary thrombosis (5 T's). AMI was a major concern in this patient, as suggested by ST-segment elevation on ECG, elevated troponin I blood level, abnormality 
of ventricular contraction, and demonstrable stenosis shown by coronary arteriography. Clearly, PE may be accompanied by myocardial ischemia, owing in part to augmented right ventricular (RV) strain.

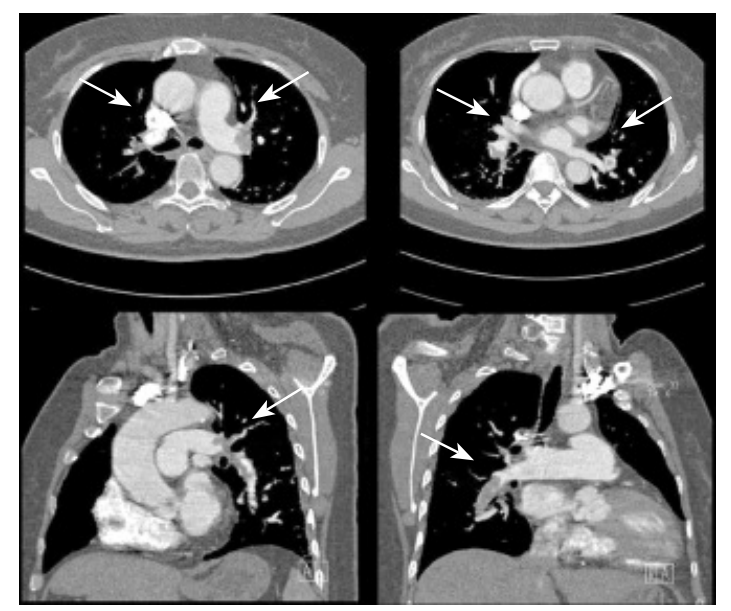

Figure 2. Filling defects on CTPA $(\rightarrow)$ involving both pulmonary arterial trunks and segmental branches, indicating bilateral pulmonary embolism.

\section{DISCUSSION}

The prognosis of patients with CA is dismal. It has been debated whether thrombolysis is warranted during CPR, given its potential benefits in instances of MPE or AMI. ${ }^{[3-5]}$ The timing of such therapy is another key issue. In a longer duration of $\mathrm{CPR}$, hemorrhagic complications and mortality may rise; but because the prognosis of patients with $\mathrm{CA}$ is poor, treatment should be aggressive to include the use of fibrinolytic agents.

We have found only a few case reports involving thrombolysis during the extended CPR of patients with MPE. These reports failed to address the impact of a hypercoagulable state under stress or during CPR. Nevertheless, Bailen et $\mathrm{a}^{[6]}$ did offer thrombolytic strategies for CA due to MPE. Fibrinolytic targeting of a single large occlusive thrombus and microvascular thrombi was one approach. Another called for reduction of blood viscosity, reversing a no-flow phenomenon to protect the brain.

After CA, intravascular fibrin deposition and thrombus formation are ongoing throughout the entire microcirculation, generating significant activation of the coagulation cascade. However, endogenous fibrinolysis is not commensurate under these circumstances. Thrombolysis may thus improve microcirculatory dynamics overall, especially in the lungs.
Factors predisposing to venous thromboembolism (VTE) include but are not limited to hip or leg fracture, major trauma/surgery, chemotherapy, malignancy, pregnancy/postpartum conditions, thrombophilia, and prolonged immobility. Two large retrospective studies have indicated that autoimmune diseases (including AIHA) are associated with hypercoagulable states or thrombophilia. As such, the risk of subsequent VTE is heightened by patient immobilization and inflammatory activity. ${ }^{[1,7]}$ Disturbed coagulation, endothelial injury, and thrombosis typically accompany severe sepsis, caused by bacterial products and proinflammatory cytokines. ${ }^{[8]}$ A thromboembolic event is more likely in the setting of AIHS or severe sepsis.

Some clinical characteristics of this patient were highly suspicious of PE at presentation (prior to CTPA confirmation), such as respiratory distress, documented CA, younger age ( $<65$ years), ECG changes, PEA episode, and echocardiogram results. All of the above are corroborated by an earlier report. ${ }^{[9]}$ The ECG changes were linked to modifications in RV pressure load, leading to alterations in right-sided cardiac conduction. A combination of sinus tachycardia and $\mathrm{T}$ wave inversion in precordial leads has recently been identified as the most common sign of RV strain. ${ }^{[10]}$ Right bundle branch block is also thought to be a marker for obstruction of the main pulmonary trunk.

Likewise, the occurrence of PEA (especially pseudoPEA) during CPR is suggestive of PE. Pseudo-PEA is usually caused by hypovolemia, tachydysrhythmias, decreased cardiac contractility or obstructions to circulation, such as tamponade, tension pneumothorax, and PE. ${ }^{[1]}$ Successful treatment depends on early identification and immediate correction of potentially reversible etiologies.

One may question whether an ultrasound study that is negative for DVT constitutes sufficient grounds for excluding PE. In reviewing the epidemiology of DVT and PE, Henzler et $\mathrm{al}^{[12]}$ found that DVT of the lower limbs could not be documented in about 30\% of patients with PE. With respect to exclusion of PE based on hemorrhage, a recent report further showed that the risk of DVT or PE did not decline with acquired coagulopathy. ${ }^{[13]}$ However, it was concluded that the utility of routine pharmacologic thromboprophylaxis for these patients remained uncertain.

As current guidelines for antithrombotic therapy and prevention of thrombosis specify, decisions on prophylaxis in nonsurgical patients should be made after considering risk factors for thrombosis and bleeding, 
clinical context, and values/preferences of patients. ${ }^{[14]}$ Low-molecular weight heparin or unfractionated heparin may be prescribed for prophylaxis or treatment of VTE. However, thrombotic and bleeding risks may coexist, as in our patient. We did not initiate antithrombotic therapy early-on (in ED) due to active gastrointestinal bleeding.

Compared with other thrombolytic agents, such as streptokinase, urokinase, tenecteplase and reteplase, alteplase is the most widely used, based on the proven benefit of a relatively brief infusion. This agent is indicated for treatment of MPE accompanied by unstable hemodynamic status. ${ }^{[15,16]}$

In conclusion, a medical history of autoimmune disease poses a risk of PE, and potential etiologies of $\mathrm{CA}$ (such as this) should be investigated. A therapeutic strategy that utilizes alteplase may be initiated early during cardiopulmonary resuscitation, once there is presumptive evidence of PE. Further trials are needed in this setting to study patients with hypercoagulable states.

\section{Funding: None.}

Ethical approval: Not needed.

Conflicts of interest: No conflicts of interest related to this report. Contributors: Gao JP proposed the study. All authors contributed to the design and interpretation of the study, and approved the final manuscript.

\section{REFERENCES}

1 Zoller B, Li X, Sundquist J, Sundquist K. Risk of pulmonary embolism in patients with autoimmune disorders: a nationwide follow-up study from Sweden. Lancet 2012; 379: 244-249.

2 Vanden Hoek TL, Morrison LJ, Shuster M, Donnino M, Sinz E, Lavonas EJ, et al. Part 12: cardiac arrest in special situations: 2010 American Heart Association Guidelines for Cardiopulmonary Resuscitation and Emergency Cardiovascular Care. Circulation 2010; 122: S829-S861.

3 Bottiger BW, Arntz HR, Chamberlain DA, Bluhmki E, Belmans $\mathrm{A}$, Danays T, et al. Thrombolysis during resuscitation for out-ofhospital cardiac arrest. N Engl J Med 2008; 359: 2651-2662.
4 Spohr F, Bottiger BW. Thrombolytics in CPR. Current advantages in cardiopulmonary resuscitation. Minerva Anestesiol 2005; 71: 291-296.

5 Logan JK, Pantle H, Huiras P, Bessman E, Bright L. Evidencebased diagnosis and thrombolytic treatment of cardiac arrest or periarrest due to suspected pulmonary embolism. Am J Emerg Med 2014; 32: 789-796.

6 Bailen MR, Cuadra JA, Aguayo de Hoyos E. Thrombolysis during cardiopulmonary resuscitation in fulminant pulmonary embolism: a review. Crit Care Med 2001; 29: 2211-2219.

7 Ramagopalan SV, Wotton CJ, Handel AE, Yeates D, Goldacre MJ. Risk of venous thromboembolism in people admitted to hospital with selected immune-mediated disease: record-linkage study. BMJ Med 2011; 9: 1.

8 van der Poll T, Levi M. Crosstalk between inflammation and coagulation: the lessons of sepsis. Curr Vasc Pharmacol 2012; 10: 632-638.

9 Flores J, de Tena JG, de Pablo R, Daguerre M. Successful outcome of cardiopulmonary arrest with systemic thrombolysis. Eur J Intern Med 2008; 19: e38-e39.

10 Abarca E, Baddi A, Manrique R. ECG manifestations in submassive and massive pulmonary embolism. Report of 4 cases and review of literature. J Electrocardiol 2014; 47: 75-79.

11 Mehta C, Brady W. Pulseless electrical activity in cardiac arrest: electrocardiographic presentations and management considerations based on the electrocardiogram. Am J Emerg Med 2012; 30: 236-239.

12 Henzler T, Schoenberg SO, Schoepf UJ, Fink C. Diagnosing acute pulmonary embolism: systematic review of evidence base and cost-effectiveness of imaging tests. J Thorac Imaging 2012; 27: 304-314.

13 Ho KM, Tan JA. Can the presence of significant coagulopathy be useful to exclude symptomatic acute pulmonary embolism? Anaesth Intensive Care 2013; 41: 322-327.

14 Kahn SR, Lim W, Dunn AS, Cushman M, Dentali F, Akl EA, et al. Prevention of VTE in nonsurgical patients: Antithrombotic Therapy and Prevention of Thrombosis, 9th ed: American College of Chest Physicians Evidence-Based Clinical Practice Guidelines. Chest 2012; 141: e195S-e226S.

15 Pedley DK, Morrison WG. Role of thrombolytic agents in cardiac arrest. Emerg Med J 2006; 23: 747-752.

16 Tapson VF. Thrombolytic therapy for acute pulmonary embolism. Semin Thromb Hemost 2013; 39: 452-458.

Received July 8, 2014 Accepted after revision January 29, 2015 